\title{
WEAK AND CYCLIC AMENABILITY FOR NON-COMMUTATIVE BANACH ALGEBRAS
}

\author{
by NIELS GRØNBÆK
}

(Received 6th August 1990)

\begin{abstract}
This paper is concerned with two notions of cohomological triviality for Banach algebras, weak amenability and cyclic amenability. The first is defined within Hochschild cohomology and the latter within cyclic cohomology. Our main result is that $H_{\lambda}^{1}(\mathscr{F})=H_{\lambda}^{1}(\mathscr{A}) \times H_{\lambda}^{1}(\mathscr{D})$, where $\mathscr{F}$ is a Banach algebraic free product of two Banach algebras $\mathscr{A}$ and $\mathscr{B}$. It follows that cyclic amenability is preserved under the formation of free products.
\end{abstract}

1980 Mathematics subject classification (1985 Revision): $46 \mathrm{H} 20$

\section{Introduction}

The notion of weak amenability was introduced in Bade et al. [1] as a concept for commutative Banach algebras: A commutative Banach algebra $\mathscr{A}$ is called weakly amenable if there are no non-zero bounded derivations into any symmetric Banach $\mathscr{A}$ module (symmetric meaning that the left and right module multiplications agree). In the same paper the authors showed that one only has to consider the module $\mathscr{A}^{*}$, the dual space of $\mathscr{A}$ equipped with the canonical module structure. Noting that a commutative Banach algebra has no non-zero inner derivations into its dual module, we then get that $\mathscr{A}$ is weakly amenable, if and only if $H^{1}\left(\mathscr{A}, \mathscr{A}^{*}\right)=(0)$, where $H^{1}(\cdot, \cdot)$ denotes the first Hochschild-Johnson cohomology group. Further investigations of weak amenability for commutative Banach algebras were made in Grønbæk [5] where a characterization is given in terms of the short exact sequence

$$
0 \rightarrow K \rightarrow \mathscr{A}^{*} \hat{\otimes} \mathscr{A} \stackrel{\pi}{\rightarrow} \rightarrow 0,
$$

where $\mathscr{A}^{*}=\mathscr{A} \oplus \mathbb{C}$ and $\pi(\alpha \otimes a)=\alpha a\left(\alpha \in \mathscr{A}^{*}, a \in \mathscr{A}\right)$.

In [8] Johnson uses the condition $H^{1}\left(\mathscr{A}, \mathscr{A}^{*}\right)=(0)$ as a definition of weak amenability for non-commutative Banach algebras and it is shown that $L^{1}(G)$ is weakly amenable, when $G$ is a SIN-group. Recently Johnson [9] has proved that $L^{1}(G)$ is weakly amenable for every locally compact group.

It is also known that all $C^{*}$-algebras are weakly amenable (Haagerup [7]).

It is often useful to restrict one's attention to derivations satisfying the property 
$\langle a, D(b)\rangle=-\langle b, D(a)\rangle(a, b \in \mathscr{A})$. (Here and throughout $\langle\cdot, \cdot\rangle$ is used for the canonical pairing of $\mathscr{A}$ and $\mathscr{A}^{*}$.) Such derivations are called cyclic. Clearly inner derivations are cyclic. The corresponding subgroup of $H^{1}\left(\mathscr{A}, \mathscr{A}^{*}\right)$ will be denoted $H_{\lambda}^{1}(\mathscr{A})$. It is the first-order cyclic cohomology group in the bounded version of the theory of cyclic cohomology developed by Connes [3] and independently from the point of view of homology by Tzygan [11]. We shall call a Banach algebra cyclicly amenable if $H_{\lambda}^{1}(\mathscr{A})=(0)$. In this paper we shall study hereditary properties of weak and cyclic amenability under the formation of some natural algebraic constructions. The main result is that

$$
H_{\lambda}^{1}(\mathscr{F})=H_{\lambda}^{1}(\mathscr{A}) \times H_{\lambda}^{1}(\mathscr{B})
$$

when $\mathscr{F}$ is a Banach algebraic free product of Banach algebras $\mathscr{A}$ and $\mathscr{B}$. In particular, if $\mathscr{A}$ and $\mathscr{B}$ are cyclicly amenable, then so is $\mathscr{F}$. It is demonstrated that $\mathscr{F}$ is never weakly amenable, regardless of $\mathscr{A}$ and $\mathscr{B}$.

The purely algebraic analogue of our result is a special case of Feigin and Tzygan [4, Theorem 3.2.1]. However, our proof is based only on the definitions of the concepts involved, whereas the proof of Feigin and Tzygan uses homological methods extensively, in particular spectral sequences, and it seems unclear whether or not it is possible to transfer the proof from algebraic cyclic homology to bounded cyclic cohomology.

In the paper by Connes [3], the point is made that through the long exact sequence connecting cyclic cohomology with Hochschild cohomology one is able to compute cyclic cohomology by computing Hochschild cohomology. The latter has the advantage of being computable in terms of the Ext-functor, so, in principle, one only has to choose an appropriate resolution. However, from the more direct approach, where one sets out to prove that a given cocycle in fact is a coboundary, it seems more natural to utilize the extra structure of cyclicity, thus obtaining results about Hochschild cohomology from results about cyclic cohomology. The main result of this paper is of this kind.

\section{Preliminaries}

This section consists mainly of definitions of the concepts involved. Throughout $\mathscr{A}$ denotes a Banach algebra and $\mathscr{A}^{*}=\mathscr{A} \oplus \mathbb{C}$ its unitization. All linear maps are continuous unless otherwise stated. The (Hochschild-Johnson) cohomology groups $H^{n}\left(\mathscr{A}, \mathscr{A}^{*}\right)$ are defined from the cochain complex

$$
\cdots \rightarrow C^{n}(\mathscr{A}) \stackrel{\delta^{n}}{\rightarrow} C^{n+1}(\mathscr{A}) \rightarrow \cdots
$$

when $C^{n}(\mathscr{A})=B L^{n+1}(\mathscr{A}, \mathbb{C}) \cong B L^{n}\left(\mathscr{A}, \mathscr{A}^{*}\right)$ and

$$
\delta^{n} T\left(a_{0}, a_{1}, \ldots, a_{n+1}\right)=\sum_{i=0}^{n}(-1)^{i} T\left(\ldots, a_{i} a_{i+1}, \ldots\right)+(-1)^{n+1} T\left(a_{n+1} a_{0}, a_{1}, \ldots, a_{n}\right)
$$


Here we have used the canonical identification of $(n+1)$-linear forms on $\mathscr{A}$ and $n$-linear maps from $\mathscr{A} \times \cdots \times \mathscr{A}$ into $\mathscr{A}^{*}$. We define the $n$-cocyles $Z^{n}\left(\mathscr{A}, \mathscr{A}^{*}\right):=\operatorname{ker} \delta^{n}$ and the $n$-coboundaries $B^{n}\left(\mathscr{A}, \mathscr{A}^{*}\right):=\operatorname{Im} \delta^{n-1}$ and set $H^{n}\left(\mathscr{A}, \mathscr{A}^{*}\right)=Z^{n}\left(\mathscr{A}, \mathscr{A}^{*}\right) / B^{n}\left(\mathscr{A}, \mathscr{A}^{*}\right)$. An $n$-cochain is called cyclic if

$$
T\left(a_{0}, \ldots, a_{n}\right)=(-1)^{n} T\left(a_{n}, a_{0}, \ldots, a_{n-1}\right)
$$

and the set of cyclic $n$-cochains is denoted $C_{\lambda}^{n}(\mathscr{A})$. It is easy to see that $C_{\lambda}^{*}(\mathscr{A})$ form a subcomplex of $C^{*}(\mathscr{A})$. The corresponding cyclic cohomology groups are denoted by $H_{\lambda}^{n}(\mathscr{A})$. Although we shall only be interested in the case $n=1$, the reader should bear in mind that the proper setting is that of cochain complexes, as described.

Definition 1.1. Let $I$ be a closed two-sided ideal of $\mathscr{A}$. We say that $I$ has the trace extension property (with respect to $\mathscr{A}$ ) if the restriction map $Z^{0}\left(\mathscr{A}, \mathscr{A}^{*}\right) \rightarrow Z^{0}\left(\mathscr{A}, I^{*}\right)$ is surjective, i.e., if every $m \in I^{*}$ satisfying $m \cdot a-a \cdot m=0(a \in \mathscr{A})$ can be extended to a trace on $\mathscr{A}$.

The following concept is important for extensions of linear maps.

Definition 1.2. Let $I$ be a closed two-sided ideal of $\mathscr{A}$. Suppose $I$ has a bounded approximate identity $\left(e_{\gamma}\right)_{\gamma \in \Gamma}$. We say that $\left(e_{\gamma}\right)_{y \in \Gamma}$ is quasi-central for $\mathscr{A}$ if $\left\|e_{\gamma} a-a e_{\gamma}\right\| \rightarrow 0$ for all $a \in \mathscr{A}$.

It is well known, see for instance Sinclair [10, Appendix 3], that, if a Banach algebra $\mathscr{A}$ has a quasi-central bounded approximate identity for its double centralizer algebra, then $\mathscr{A}$ has a quasi-central bounded approximate identity for any Banach algebra containing $\mathscr{A}$ as an ideal. Examples of Banach algebras with such quasi-central bounded approximate identities are Arens regular algebras with bounded approximate identities, in particular $C^{*}$-algebras.

Proposition 1.3. Suppose $I$ has a bounded approximate identity quasi-central for $\mathscr{A}$. Then $I$ has the trace extension property with respect to $\mathscr{A}$ and every derivation $D: I \rightarrow I^{*}$ can be lifted to a derivation $\tilde{D}: \mathscr{A} \rightarrow \mathscr{A}^{*}$.

Proof. Let $\left(e_{y}\right)_{y \in \Gamma}$ be a quasi-central bounded approximate identity. We define a family of bounded linear maps $J_{\gamma}: I^{*} \rightarrow \mathscr{A}^{*}(\gamma \in \Gamma)$ by

$$
\left\langle a, J_{\gamma}(m)\right\rangle=\left\langle e_{y} a, m\right\rangle \quad\left(a \in \mathscr{A}, m \in I^{*}\right) .
$$

By identifying $B\left(I^{*}, \mathscr{A}^{*}\right)=\left(I^{*} \widehat{\otimes} \mathscr{A}\right)^{*}$ and passing to a subnet if necessary, we may assume that the net $\left(J_{\gamma}\right)_{y \in \Gamma}$ is convergent in the weak operator topology to a bounded linear map $J: I^{*} \rightarrow \mathscr{A}^{*}$. Using the quasi-central property of $\left(e_{y}\right)_{y \in \Gamma}$ one easily checks that $J$ is a module map with the property that $J(m)$ is an extension of $m$ for each $m \in I^{*}$. 
Obviously, if $m \in Z^{0}\left(\mathscr{A}, I^{*}\right)$, then $J(m)$ is a trace. If $D: I \rightarrow I^{*}$ is a derivation we first extend to a derivation $\bar{D}: \mathscr{A} \rightarrow I^{*}$, using the standard construction $\bar{D}(a)=w^{*}-\lim _{y} D\left(e_{y} a\right)$. Then $\tilde{D}=J \bar{D}$ is the desired derivation.

We shall now define a Banach algebraic free product. It is a suitable completion of the corresponding algebraic object.

Definition 1.4. Let $\left(\mathscr{A}_{\gamma}\right)_{y \in \Gamma}$ be a family of Banach algebras. A Banach algebraic free product of $\left(\mathscr{A}_{\gamma}\right)_{\gamma \in \Gamma}$ is a Banach algebra $\mathscr{F}$ satisfying the following universal property. There are isometries $i_{\gamma}: \mathscr{A}_{\gamma} \rightarrow \mathscr{F}$ so that, whenever $\phi_{\gamma}: \mathscr{A}_{\gamma} \rightarrow \mathscr{B}$ is a family of bounded homomorphisms into a Banach algebra $\mathscr{B}$ with $\sup \left\{\left\|\phi_{\gamma}\right\| \| \gamma \in \Gamma\right\} \leqq 1$, there is a unique bounded homomorphism $\phi: \mathscr{F} \rightarrow \mathscr{B}$ so that the diagrams

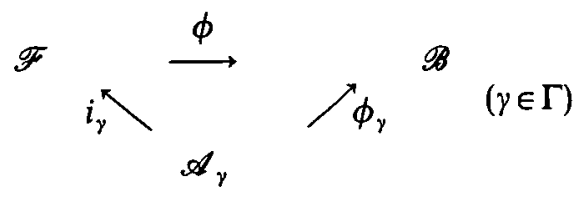

all commute.

For existence and uniqueness (up to isomorphism), see Christensen, Effros, and Sinclair [2]. We use the notation $\underset{\gamma \in \Gamma}{\hat{\imath}} \mathscr{A}_{\gamma}$ for $\mathscr{F}$.

Example 1.5. We want to recall a useful representation of Banach algebraic free products. Let $\mathscr{A}$ and $\mathscr{B}$ be two Banach algebras. Then $\mathscr{A} \mathscr{B} \mathscr{B}$ is defined as

$$
\mathscr{A} \bullet \mathscr{B}=\bigoplus_{n=1}^{\infty} A_{n} \oplus \bigoplus_{n=1}^{\infty} B_{n},
$$

where $A_{n}=\mathscr{A} \hat{\otimes} \mathscr{B} \hat{\otimes} \mathscr{A} \hat{\otimes} \mathscr{B} \cdots$ (n factors) and $B_{n}=\mathscr{B} \hat{\otimes} \mathscr{A} \hat{\otimes} \mathscr{B} \hat{\otimes} \cdots$ ( $n$ factors) as Banach spaces. Here $\oplus$ denotes $l^{1}$-direct sum. The algebra product on $\mathscr{A} \mathscr{A} \mathscr{B}$ is given by the tensor product modulo the relations $a_{1} \otimes a_{2}=a_{1} a_{2}, b_{1} \otimes b_{2}=b_{1} b_{2}\left(a_{i} \in \mathscr{A}, b_{i} \in \mathscr{B}\right.$, $i=1,2)$. For further details, see Christensen, Effros, and Sinclair [2].

In general, we define $\mathscr{A}_{1} \hat{\bullet} \cdots \mathscr{A}_{n}$ as $\left(\mathscr{A}_{1} \hat{\bullet} \cdots \hat{\bullet}_{n-1}\right) \hat{\bullet} \mathscr{A}_{n}, n \geqq 2$, and for an arbitrary family, we define

$$
\underset{\gamma \in \Gamma}{\bullet} \stackrel{\lim }{\longrightarrow} \underset{F \in \mathscr{P}_{0}(\Gamma)}{\longrightarrow}\left(\underset{\gamma \in F}{\hat{\bullet}} \mathscr{A}_{\gamma}\right),
$$

where $\mathscr{P}_{0}(\Gamma)$ is the set of finite subsets of $\Gamma$ and the direct limit is taken with respect to the inclusion order on $\mathscr{P}_{0}(F)$ and the canonical embeddings 


$$
\underset{\gamma \in F_{1}}{\bullet} \mathscr{A}_{\gamma} \hookrightarrow \underset{\gamma \in F_{2}}{\bullet} \mathscr{A}_{y}\left(F_{1} \subseteq F_{2}\right)
$$
etc.

The generic products will from here on be denoted $a_{1} b_{1} \ldots$, etc. instead of $a_{1} \otimes b_{1} \ldots$,

\section{Weak and cyclic amenability of extensions and free products}

Given a derivation $D: \mathscr{A} \rightarrow \mathscr{A}^{*}$ it is natural to ask if $D$ can be extended to a derivation $\tilde{D}: \mathscr{A}^{*} \rightarrow\left(\mathscr{A}^{*}\right)^{*}$. The answer is given by the following.

Proposition 2.1. Let $D: \mathscr{A} \rightarrow \mathscr{A}^{*}$ be a derivation. Then $D$ can be extended to a derivation $\tilde{D}: \mathscr{A}^{*} \rightarrow\left(\mathscr{A}^{*}\right)^{*}$, if and only if there is a constant $K \geqq 0$ so that

$$
\left|\sum_{i=1}^{n}\left(\left\langle a_{i}, D\left(b_{i}\right)\right\rangle+\left\langle b_{i}, D\left(a_{i}\right)\right\rangle\right)\right| \leqq K\left\|\sum_{i=1}^{n} a_{i} b_{i}\right\|
$$

$\left(a_{i}, b_{i} \in \mathscr{A}_{i} ; i=1, \ldots, n\right)$.

Proof. Suppose $D$ can be extended. Denote the extension by $\tilde{D}$. Then

$$
\begin{aligned}
\left|\sum_{i=1}^{n}\left(\left\langle a_{i}, D\left(b_{i}\right)\right\rangle+\left\langle b_{i}, D\left(a_{i}\right)\right\rangle\right)\right| & =\left|\left\langle 1, \tilde{D}\left(\sum_{i=1}^{n} a_{i} b_{i}\right)\right\rangle\right| \\
& \leqq\|1\|\|\tilde{D}\|\left\|\sum_{i=1}^{n} a_{i} b_{i}\right\| .
\end{aligned}
$$

Conversely, suppose the inequalities hold and define a linear functional by $\phi(a b)=$ $\langle a, D(b)\rangle+\langle b, D(a)\rangle(a, b \in \mathscr{A})$. The inequalities imply that $\phi$ can be extended to a bounded linear functional on $\mathscr{A}$. We identify $\left(\mathscr{A}^{*}\right)^{*}$ with $\mathbb{C} \times \mathscr{A}^{*}$. Put

$$
\tilde{D}(\lambda 1+a)=(\phi(a), D(a)) \quad(\lambda \in \mathbb{C}, a \in \mathscr{A})
$$

A routine calculation verifies that $\tilde{D}$ is a bounded derivation, extending $D$.

Corollary 2.2. If $\mathscr{A}$ has a bounded two-sided approximate unit, then every derivation $D: \mathscr{A} \rightarrow \mathscr{A}^{*}$ can be extended to a derivation $\tilde{D}: \mathscr{A}^{*} \rightarrow\left(\mathscr{A}^{*}\right)^{*}$. Each cyclic derivation can be extended.

Proof. Let $\left(e_{i}\right)_{i \in I}$ be a bounded two-sided approximate identity for $\mathscr{A}$ and let $a_{1}, \ldots, a_{n}, b_{1}, \ldots, b_{n} \in \mathscr{A}$. Then 


$$
\begin{aligned}
\mid \sum_{1}^{n}\left(\left\langle a_{k}, D\left(b_{k}\right)\right\rangle+\left\langle b_{k}, D\left(a_{k}\right)\right\rangle\right) & =\lim _{i}\left|\sum_{1}^{n}\left(\left\langle e_{i} a_{k}, D\left(b_{k}\right)\right\rangle+\left\langle b_{k} e_{i}, D\left(a_{k}\right)\right\rangle\right)\right| \\
& =\lim _{i}\left|\sum_{1}^{n}\left\langle e_{i}, D\left(a_{k} b_{k}\right)\right\rangle\right| \\
& \leqq \limsup _{i}\left\|e_{i}\right\|\|D\|\left\|\sum_{1}^{n} a_{k} b_{k}\right\| .
\end{aligned}
$$

Alternatively, invoke Proposition 1.3.

Remark. As mentioned in the introduction a $C^{*}$-algebra $\mathscr{A}$ is weakly amenable. The proof of this uses deep and fundamental properties of $C^{*}$-algebras, notably the Grothendieck inequality. However, it is easy to see that all derivations are cyclic. By Corollary 2.2 we may assume that $\mathscr{A}$ is unital. Hence we must prove that $\langle 1, D(a)\rangle=0$ $(a \in \mathscr{A})$. If $a$ is normal this is an obvious statement about commutative $C^{*}$-algebras, so it clearly holds for any $a \in \mathscr{A}$. A similar reasoning applies to discrete convolution algebras.

Example 2.3. Let $\mathscr{A}^{2}=(0)$ and choose $f \in \mathscr{A}^{*} \backslash(0)$. It is immediate to verify that the map $D: \mathscr{A} \rightarrow \mathscr{A}^{*}$, given by $D a=f(a) f(a \in \mathscr{A})$, is a derivation, and since $\langle a, D(b)\rangle+\langle b, D(a)\rangle=2 f(a) f(b)$ it is clear that $D$ cannot be extended to $\mathscr{A}^{*}$.

We now turn to a description of hereditary properties of weak and cyclic amenability under extensions. Consider the short exact sequence of Banach algebras and continuous homomorphisms

$$
\stackrel{j}{j} \stackrel{\theta}{\rightarrow} \stackrel{\mathscr{A}}{\rightarrow} \mathscr{B} \rightarrow 0
$$

We shall identify $\mathscr{I}$ with a closed two-sided ideal in $\mathscr{A}$. Since arguments for heredity of weak and cyclic amenability are parallel we shall give them for weak amenability and just insert the different phrasing needed for the cyclic amenability case in square brackets $[\cdots]$.

Proposition 2.4. Consider the short exact sequence $0 \rightarrow \mathscr{I} \stackrel{\dot{S}}{\rightarrow} \stackrel{\mathscr{A}}{\rightarrow} \mathscr{B} \rightarrow 0$.

(i) If $\mathscr{B}$ is cyclicly amenable, then $\mathscr{I}$ has the trace extension property.

(ii) If $\mathscr{A}$ is weakly [cyclicly] amenable and $\mathscr{I}$ has the trace extension property, then $\mathscr{B}$ is weakly [cyclicly] amenable.

(iii) If $\mathscr{A}$ is weakly [cyclicly] amenable and $\mathscr{I}$ has a quasi-central bounded approximate identity for $\mathscr{A}$, then $\mathscr{I}$ is weakly [cyclicly] amenable. 
(iv) If $\mathscr{B}$ is weakly amenable and $\mathscr{I}$ is weakly amenable $[\mathscr{I}$ is cyclicly amenable and $\left.\left(\mathscr{I}^{2}\right)^{-}=\mathscr{I}\right]$, then $\mathscr{A}$ is weakly [cyclicly] amenable.

Proof. Consider the short exact sequence of cochain complexes

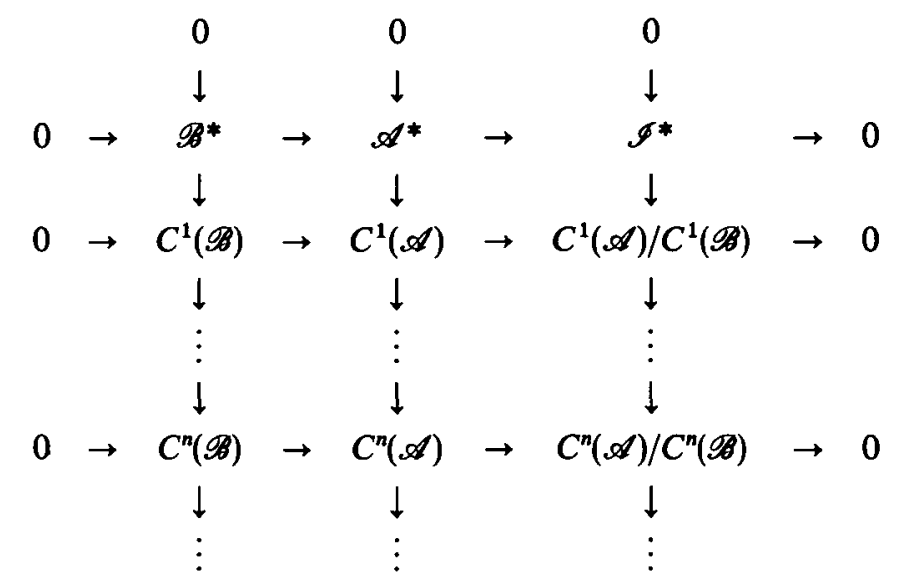

Taking the long exact sequence of cohomology we obtain

$$
\cdots \rightarrow H^{0}\left(\mathscr{A}, \mathscr{A}^{*}\right) \rightarrow H^{0}\left(\mathscr{A}, \mathscr{I}^{*}\right) \rightarrow H^{1}\left(\mathscr{B}, \mathscr{B}^{*}\right) \rightarrow H^{1}\left(\mathscr{A}, \mathscr{A}^{*}\right) \rightarrow \cdots
$$

from which (i) and (ii) follow. [The corresponding cyclic cochain complexes give the cyclic version.] Note that this argument is purely algebraic, using only exactness and the canonical identification $\mathscr{I}^{*} \sim \mathscr{A}^{*} / \mathscr{B}^{*}$.

(iii). By Proposition 1.3, any bounded [cyclic] derivation $D: \mathscr{I} \rightarrow \mathscr{I}^{*}$ can be lifted to a [cyclic] derivation $\bar{D}: \mathscr{A} \rightarrow \mathscr{A}^{*}$. From this, the claim follows immediately.

(iv). Let $D: \mathscr{A} \rightarrow \mathscr{A}^{*}$ be a [cyclic] derivation. Since $\mathscr{I}$ is weakly [cyclicly] amenable the derivation $\imath^{*} D i$ is inner, say generated by $f \in \mathscr{I}^{*}$. Extend $f$ to an element in $\mathscr{A}^{*}$ and let $\delta: \mathscr{A} \rightarrow \mathscr{A}^{*}$ be the corresponding inner derivation. We then have $(D-\delta) \mathscr{I} \subseteq \mathscr{I}^{\perp}$ so that $(D-\delta)\left(\mathscr{I}^{2}\right)=(0)$. Suppose $\left(\mathscr{I}^{2}\right)^{-} \neq \mathscr{I}$ and take $f \in \mathscr{I}^{*} \backslash(0)$ with $f\left(\mathscr{I}^{2}\right)=(0)$. As in Example 2.5 one checks that $D(x)=f(x) f(x \in \mathscr{F})$ defines a non-inner derivation. Hence since $\mathscr{I}$ is weakly amenable we have [by assumption] $\left(\mathscr{I}^{2}\right)^{-}=\mathscr{I}$, so that $(D-\delta)(\mathscr{I})=$ (0). Similarly, $(D-\delta)(\mathscr{A}) \subseteq \mathscr{I}^{\perp}$. Using the canonical isomorphism $\mathscr{B}^{*} \cong \mathscr{I}^{\perp}$, we see that $(D-\delta)$ drops to a [cyclic] derivation from $\mathscr{B}$ into $\mathscr{B}^{*}$. Since $\mathscr{B}$ is weakly [cyclicly] amenable it follows that $(D-\delta)$ is inner, proving that $D$ is inner.

Example 2.5. One may ask, whether the condition $\left(\mathscr{I}^{2}\right)^{-}=\mathscr{I}$ is necessary in the cyclic version of Proposition 2.6(iv). The following example shows that some condition is needed. Let $\mathscr{R} \neq(0)$ be a Banach algebra with zero algebra product. Then $\mathscr{R}$ is cyclicly amenable if and only if $\operatorname{dim} \mathscr{R}=1$. To see this, first note that any linear map $D: \mathscr{R} \rightarrow \mathscr{R}^{*}$ satisfies the derivation rule. If we define

$$
\langle a, T b\rangle=\langle a, D(b)\rangle-\langle b, D(a)\rangle \quad(a, b \in \mathscr{R})
$$


we get a cyclic derivation. If $\operatorname{dim} \mathscr{R} \geqq 2$ we may choose $D$ such that $T$ is non-zero and hence non-inner, since $\mathscr{R}$ is commutative. On the other hand, if $\mathscr{R}=\mathbb{C}$ with zero algebra product, then 0 is the only cyclic 1-cochain. It follows that the split extension given by

$$
0 \rightarrow \mathscr{R} \rightarrow \mathscr{R} \oplus \mathscr{R} \rightarrow \mathscr{R} \rightarrow 0
$$

is not cyclicly amenable.

Now let $\mathscr{F}$ be a Banach algebraic free product of two Banach algebras $\mathscr{A}$ and $\mathscr{B}$ and let $\mathscr{F}_{0}$ denote the corresponding algebraic free product, consisting of finite sums of algebraic tensors in the description from Example 1.5. Using the well-known identification of derivations with homomorphisms into singular extensions, it is clear that in the diagram

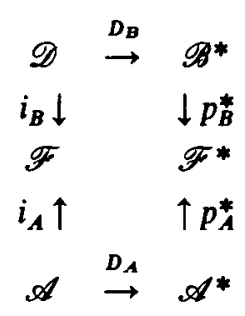

each pair of derivations $\left(D_{A}, D_{B}\right)$ can be lifted to a (unique) derivation $\Delta: \mathscr{F}_{0} \rightarrow \mathscr{F}^{*}$. The maps $p_{A}$ and $p_{B}$ are the retractions obtained from lifting the identity isomorphisms of $\mathscr{A}$ and $\mathscr{B}$.

The derivation $\Delta$ has the form

$$
\Delta\left(a_{1} b_{1} \cdots\right)=p_{A}^{*} D_{A}\left(a_{1}\right) b_{1} \cdots+a_{1} p_{B}^{*} D_{B}\left(b_{1}\right) \cdots+\cdots
$$

Since $p_{A}^{*}\left(\mathscr{A}^{*}\right)$ is in the annihilator of the canonical complement of $i_{A}(\mathscr{A})$ and similarly for $p_{B}^{*}\left(\mathscr{B} B^{*}\right)$, the derivation $\Delta$ is zero on the canonical complement of $i_{A}(\mathscr{A}) \oplus i_{B}(\mathscr{B})$, so $\Delta$ is obviously continuous with $\|\Delta\|=\max \left\{\left\|D_{A}\right\|,\left\|D_{B}\right\|\right\}$. The extension by continuity to all of $\mathscr{F}$ completes the diagram.

The canonical map of cochain complexes given by $\imath: C^{n}(\mathscr{F}) \rightarrow C^{n}(\mathscr{A}) \times C^{n}(\mathscr{B})$, where $i(T)=\left(T \circ\left(i_{A} \times \cdots \times i_{A}\right), T \circ\left(i_{B} \times \cdots \times i_{B}\right)\right)$ induces a map of cohomology $i^{*}: H^{n}(\mathscr{F}) \rightarrow$ $H^{n}(\mathscr{A}) \times H^{n}(\mathscr{B})$.

As pointed out above, for $n=1$ this map is surjective. However, it is never injective as the following example shows.

Example 2.6. We identify $\mathscr{A}$ and $\mathscr{B}$ with their canonical images in $\mathscr{F}$. Choose a functional $f \in \mathscr{F}^{*}$ such that $f(a b-b a) \neq 0$ for some $a \in \mathscr{A}, b \in \mathscr{B}$. Let $D_{A}: \mathscr{A} \rightarrow \mathscr{F} *$ be the inner derivation $\mathscr{D}_{A}(a)=f \cdot a-a \cdot f$ and let $\mathscr{D}_{B}: \mathscr{B} \rightarrow \mathscr{F}^{*}$ be identically zero. Due to the universal property of $\mathscr{F}_{0}$, there is a (unique) derivation $\Delta: \mathscr{F}_{0} \rightarrow \mathscr{F}^{*}$ such that $\Delta(b)=0$ and $\Delta(a)=f \cdot a-a \cdot f$. If we choose $f$ to be supported on $A_{2}$ (cf. Example 1.5) we get the identities 


$$
\begin{aligned}
\Delta(a) & =f \cdot a \\
\left.\Delta\right|_{A_{n} \oplus B_{n}} & =0 \quad \text { for } n \geqq 4 \\
\Delta\left(a_{1} b a_{2}\right) & =a_{1} b \cdot f \cdot a_{2} \\
\Delta\left(b_{1} a b_{2}\right) & =b_{1} \cdot f \cdot a b_{2} \\
\Delta(a b) & =f \cdot a b \\
\Delta(b a) & =b \cdot f \cdot a
\end{aligned}
$$

from which it follows that $\|\Delta\| \leqq\|f\|$, so that $\Delta$ can be extended to a continuous derivation on $\mathscr{F}$. Since $\langle a, \Delta(b)\rangle \neq-\langle b, \Delta(a)\rangle$, the derivation $\Delta$ cannot be inner. But $\left(i_{A}^{*} \Delta \circ i_{A}, i_{B}^{*} \Delta \circ i_{B}\right)$ is clearly a pair of inner derivations.

Nevertheless, if we restrict to cyclic cohomology we have:

Theorem 2.7. Let $\mathscr{A}$ and $\mathscr{B}$ be two Banach algebras and let $\mathscr{F}$ be a Banach algebraic free product of $\mathscr{A}$ and $\mathscr{B}$. Then $i^{*}: H_{\lambda}^{1}(\mathscr{F}) \rightarrow H_{\lambda}^{1}(\mathscr{A}) \times H_{\lambda}^{1}(\mathscr{B})$ is an isomorphism.

Proof. It is clear that $\imath^{*}$ takes cyclic cochains to pairs of cyclic cochains. We identify $\mathscr{A}$ and $\mathscr{B}$ with their canonical images in $\mathscr{F}$. First we prove that $\imath^{*}$ is onto. This amounts to showing that, if $D_{A}: \mathscr{A} \rightarrow \mathscr{A}^{*}$ and $D_{B}: \mathscr{B} \rightarrow \mathscr{B}^{*}$ are cyclic, then so is the lift $\Delta: \mathscr{F} \rightarrow \mathscr{F}^{*}$. We shall use the representation of $\mathscr{F}$ described in Example 1.5. The symbol $\Theta$ will denote taking complements with respect to the defining $l^{1}$-sum. Let $a_{1} b_{1} \cdots \in \mathscr{F} \ominus(\mathscr{A}+\mathscr{B})$ be an elementary tensor, and let $\omega \in \mathscr{F}$ be arbitrary. Then

$$
\begin{aligned}
\left\langle\omega, \Delta\left(a_{1} b_{1} \ldots\right)\right\rangle & =\left\langle\omega, \Delta\left(a_{1}\right) \cdot b_{1} \ldots\right\rangle+\left\langle\omega, a_{1} \cdot \Delta\left(b_{1}\right) \ldots\right)+\cdots \\
& =\left\langle b_{1} \ldots \omega, \Delta\left(a_{1}\right)\right\rangle+\left\langle\ldots \omega a_{1}, \Delta\left(b_{1}\right)\right\rangle+\cdots \\
& =\left\langle p_{A}\left(b_{1} \ldots \omega\right), D_{A}\left(a_{1}\right)\right\rangle+\left\langle p_{B}\left(\ldots \omega a_{1}\right), D_{B}\left(b_{1}\right)\right\rangle+\cdots \\
& =0
\end{aligned}
$$

and similarly if the product starts with a factor from $\mathscr{B}$.

Hence for $\omega_{i}=a_{i}+b_{i}+\xi_{i}$, where $a_{i} \in \mathscr{A}, b_{i} \in \mathscr{B}, \xi_{i} \in \mathscr{F} \ominus(\mathscr{A}+\mathscr{B}), i=1,2$, we have

$$
\begin{aligned}
\left\langle\omega_{1}, \Delta\left(\omega_{2}\right)\right\rangle & =\left\langle p_{A}\left(a_{1}+b_{1}+\xi_{1}\right), D_{A}\left(a_{2}\right)\right\rangle+\left\langle p_{B}\left(a_{1}+b_{1}+\xi_{1}\right), D_{B}\left(b_{2}\right)\right\rangle \\
& =\left\langle a_{1}, D_{A}\left(a_{2}\right)\right\rangle+\left\langle b_{1}, D_{B}\left(b_{2}\right)\right\rangle \\
& =-\left\langle a_{2}, D_{A}\left(a_{1}\right)\right\rangle-\left\langle b_{2}, D_{B}\left(b_{1}\right)\right\rangle
\end{aligned}
$$




$$
=-\left\langle\omega_{2}, \Delta\left(\omega_{1}\right)\right\rangle \text {. }
$$

To finish, we must show that, if $\Delta: \mathscr{F} \rightarrow \mathscr{F}^{*}$ is cyclic and $t_{A}^{*} \Delta i_{A}$ and $i_{B}^{*} \Delta l_{B}$ are both inner, then $\Delta$ is inner.

Proposition 2.8. Suppose that $\Delta: \mathscr{F} \rightarrow \mathscr{F}^{*}$ is a cyclic derivation and that $i_{A}^{*} \Delta l_{A}$ and $i_{B}^{*} \Delta i_{B}$ are inner derivations, generated by $f_{A} \in \mathscr{A}^{*}$ and $f_{B} \in \mathscr{B} *$ respectively. Then $\Delta$ is inner by $g \in \mathscr{F}^{*}$ with

$$
\|g\| \leqq\left\|f_{A}\right\| \vee\left\|f_{B}\right\| \vee\|\Delta\|
$$

Proof. The proof proceeds through three lemmas:

Lemma 2.9. Let $\Delta: \mathscr{F} \rightarrow \mathscr{F}^{*}$ be a derivation such that $i_{A}^{*} \Delta t_{A}$ is inner by $f_{A}$. Then there is $f_{X} \in \mathscr{F}^{*}$ such that $\Delta \circ i_{A}$ is inner by $f_{X}$ and $\left\|f_{X}\right\| \leqq\|\Delta\| \vee\left\|f_{A}\right\|$.

Proof. Let $t \in \mathscr{F} \ominus \mathscr{A}$ be a (non-zero) elementary tensor. Then $t$ has one of the forms

(i) $t \in \cup B_{n}$

(ii) $t=\xi y$, where $\xi \in \mathscr{A}, y \in \cup B_{n}$.

Here $B_{n}=\mathscr{B} \widehat{\otimes} \mathscr{A} \otimes \mathscr{B} \otimes \cdots$ in the decomposition of $\mathscr{F}$.

Define

$$
f(t)= \begin{cases}0 & \text { if } t \text { is of type (i) } \\ \langle y, \Delta(\xi)\rangle & \text { if } t \text { is of type (ii). }\end{cases}
$$

By the defining property of the projective tensor product this gives a well-defined linear form on $\mathscr{F} \ominus \mathscr{A}$ with $\|f\| \leqq\|\Delta\|$. Suppose $t$ is of type (i) and let $a \in \mathscr{A}$. Then

$$
\begin{aligned}
\langle t, \Delta(a)\rangle & =f(a t) \\
& =f(a t-t a),
\end{aligned}
$$

since $t a$ is of type (i). If $t$ is of type (ii), write $t=\xi y$. Then

$$
\begin{aligned}
\langle t, \Delta(a)\rangle & =\langle\xi y, \Delta(a)\rangle \\
& =\langle y, \Delta(a \xi)\rangle-\langle y a, \Delta(\xi)\rangle \\
& =f(a \xi y-y a \xi)-f(\xi y a-y a \xi) \\
& =f(a t-t a),
\end{aligned}
$$


since $t$ and $y a$ are of type (i). Let $f_{A}=f_{A} \oplus f$. Then $f_{X}$ is the required functional.

Lemma 2.10. Suppose $\Delta: \mathscr{F} \rightarrow \mathscr{F}^{*}$ is cyclic, that $\Delta \circ i_{B}=0$, and that $\Delta \circ l_{A}$ is inner by some $f \in \mathscr{F}^{*}$. Then $f$ satisfies

$$
\sum_{i=1}^{n} f\left(a_{i} b_{i} a_{i+1} \ldots b_{n} a_{1} \ldots b_{i-1}\right)=\sum_{i=1}^{n} f\left(b_{i} a_{i+1} \ldots b_{n} a_{1} \ldots a_{i}\right)
$$

for any $a_{1}, \ldots, a_{n} \in \mathscr{A}, b_{1}, \ldots, b_{n} \in \mathscr{B}$.

Proof. Since $\Delta$ is cyclic and $\Delta(\mathscr{B})=\{0\}$ we have

$$
\begin{aligned}
0 & =\left\langle b_{n}, \Delta\left(a_{1} b_{1} \ldots a_{n}\right)\right\rangle \\
& =\sum_{i=1}^{n}\left\langle b_{n}, \ldots \Delta\left(a_{i}\right) \ldots\right\rangle \\
& =\sum_{i=1}^{n}\left\langle b_{n}, \ldots b_{i-1} \cdot\left[f \cdot a_{i}-a_{i} \cdot f\right] \cdot b_{i} \ldots\right\rangle \\
& =\sum_{i=1}^{n}\left[\left\langle a_{i} b_{i} \ldots b_{n} a_{1} \ldots b_{i-1}, f\right\rangle-\left\langle b_{i} \ldots a_{n} b_{n} a_{1} \ldots a_{i}, f\right\rangle\right]
\end{aligned}
$$

which exactly is the statement of the lemma.

Lemma 2.11. Suppose that $f \in \mathscr{F}^{*}$ and $\Delta$ satisfy the conditions of Lemma 2.10. Then there are $g_{A}, g_{B} \in \mathscr{F}^{*}$ so that

(i) $f=g_{A}+g_{B}$

(ii) $g_{A} \cdot a-a \cdot g_{A}=g_{B} \cdot b-b \cdot g_{B}=0 \quad(a \in \mathscr{A}, b \in \mathscr{B})$

and $\left\|g_{B}\right\| \leqq\|\Delta\| \vee\left\|\left.f\right|_{\mathscr{A}+\mathscr{B}}\right\|$.

Proof. Put $X=\bigoplus_{i=2}^{\infty} A_{i}$ and $Y=\bigoplus_{i=2}^{\infty} B_{i}$ where $A_{i}$ and $B_{i}$ are the subspaces in the decomposition of $\mathscr{F}$ and let $\pi_{X}$ and $\pi_{Y}$ denote the corresponding projections with respect to the direct sum decomposition $\mathscr{F}=\mathscr{A} \oplus \mathscr{B} \oplus X \oplus Y$. Let $\sigma: X \oplus Y \rightarrow X \oplus Y$ be the "flip" induced by $\sigma\left(v_{1} v_{2} \ldots v_{n}\right)=v_{2} v_{3} \ldots v_{n} v_{1}$, where $v_{1} \ldots v_{n}$ is an elementary tensor in $A_{n} \oplus B_{n}$. Then $\sigma$ is a contraction. Let $\sigma_{n}$ be the restriction to $A_{n} \oplus B_{n}$. Then $\sigma_{2 n}: A_{2 n} \oplus$ $B_{2 n} \rightarrow A_{2 n} \oplus B_{2 n}$ is an isometry of order $2 n$. From the easily verifiable identities

$$
\left(I-\sigma_{2 n}\right) \sum_{i=0}^{2 n-1} \sigma_{2 n}^{i}=0
$$

and 


$$
\left(I-\sigma_{2 n}\right) \sum_{i=1}^{2 n-1} i \sigma_{2 n}^{-(i+1)}=n I-\sum_{i=0}^{2 n-1} \sigma_{2 n}^{i}
$$

it follows that $\operatorname{Im}\left(I-\sigma_{2 n}\right)=\operatorname{ker}\left(\sum_{i=0}^{2 n-1} \sigma_{2 n}^{i}\right)$. In particular $I-\sigma_{2 n}$ has closed range in $A_{2 n} \oplus B_{2 n}$. Define $h_{B}$ on $A_{2 n} \oplus B_{2 n}$ by

$$
h_{B}\left(1-\sigma_{2 n}\right)=f\left(1-\sigma_{2 n}\right) \pi_{X} .
$$

This is well defined. Suppose that $\left(1-\sigma_{2 n}\right)(x+y)=0$ for some $x \in A_{2 n}, y \in B_{2 n}$. Since $\sigma_{2 n}$ interchanges $A_{2 n}$ and $B_{2 n}$, we have $x=\sigma_{2 n}(y)$ and $y=\sigma_{2 n}(x)$, so that $\sigma_{2 n}^{2 n}(x)=x$ and $\sigma_{2 n}^{2}(y)=y$. Therefore

$$
\begin{aligned}
f\left(I-\sigma_{2 n}\right) \pi_{X}(x+y) & =f\left(I-\sigma_{2 n}\right) x \\
& =\frac{1}{n} \sum f\left(\left(\sigma^{2 i}-\sigma^{2 i+1}\right) x\right) \\
& =0,
\end{aligned}
$$

where the last equality follows from the conclusion of Lemma 2.12. Since $I-\sigma_{2 n}$ has closed range we may extend $h_{B}$ to a bounded linear functional on $A_{2 n} \oplus B_{2 n}$. Define $h_{A}$ on $A_{2 n}+B_{2 n}$ by means of the equation $f=h_{A}+h_{B}$. On $B_{2 n+1}$ define $h_{B}=h_{B} \sigma_{2 n+1}$. This is possible, because $\sigma_{2 n+1}$ maps $B_{2 n+1}$ into $A_{2 n}$ and $h_{B}$ has already been defined on $A_{2 n}$. Similarly, define $h_{A}$ on $A_{2 n+1}$ by $h_{A}=h_{A} \sigma_{2 n+1}$. On $\mathscr{A} \oplus \mathscr{B}$ put $h_{B}=f$ and $h_{\mathrm{A}}=0$. Finally, define $h_{A}$ and $h_{B}$ on all of $\sum_{i=1}^{\infty} A_{i} \oplus B_{i}$ (algebraic direct sum) by means of the equation $f=h_{A}+h_{B}$. So far we have obtained (i) and (ii) algebraically on $\sum_{i=1}^{\infty} A_{i} \oplus B_{i}$. Note that the solution is uniquely determined by the restrictions $\left.h_{B}\right|_{A_{2 n} \oplus B_{2 n}}(n \in \mathbf{N})$. We now replace $h_{B}$ on $A_{2 n} \oplus B_{2 n}$ by

$$
g_{B}=h_{B}-\frac{1}{2 n} \sum_{i=1}^{2 n} h_{B} \sigma_{2 n}^{i}
$$

We have clearly $\left\langle\omega_{1}, \Delta\left(\omega_{2}\right)\right\rangle=h_{B}\left(\omega_{2} \omega_{1}-\omega_{1} \omega_{2}\right)$ for all algebraic tensors $\omega_{1}, \omega_{2} \in \mathscr{F}$. Let $t=v_{1} v_{2} \ldots v_{2 n}$ be an elementary tensor in $A_{2 n} \oplus B_{2 n}$. Then

$$
\begin{aligned}
\left|g_{B}(t)\right| & =\frac{1}{2 n}\left|\sum_{i=1}^{2 n} h_{B}\left(1-\sigma_{2 n}^{i}\right) t\right| \\
& =\frac{1}{2 n}\left|\sum_{i=1}^{2 n} h_{B}\left(v_{1} \ldots v_{2 n}-v_{i+1} \ldots v_{2 n} v_{1} \ldots v_{i}\right)\right| \\
& \leqq \frac{1}{2 n} \sum_{i=1}^{2 n}\left|\left\langle v_{i+1} \ldots v_{2 n}, \Delta\left(v_{1} \ldots v_{i}\right)\right\rangle\right|
\end{aligned}
$$




$$
\begin{aligned}
& \leqq \frac{1}{2 n} \sum_{i=1}^{2 n}\|\Delta\|\left\|v_{1}\right\| \cdots\left\|v_{2 n}\right\| \\
& =\|\Delta\|\|t\| .
\end{aligned}
$$

so that $\left\|g_{B}\right\| \leqq\|\Delta\|$. Since $g_{B}\left(1-\sigma_{2 n}\right)=h_{B}\left(1-\sigma_{2 n}\right)$ we see that $g_{B}$ satisfies the properties needed.

Proof of Proposition 2.8 (continued). Let $\Delta: \mathscr{F} \rightarrow \mathscr{F}^{*}$ be the derivation. By Lemma $2.9 \Delta \circ i_{B}$ is inner by $f_{Y} \in \mathscr{F}^{*}$. Let $\delta: \mathscr{F} \rightarrow \mathscr{F}^{*}$ be the inner derivation generated by $f_{Y}$. Then $\Delta^{\prime}=\Delta-\delta$ satisfies the hypothesis of Lemma 2.10 with $f=f_{A}-f_{Y}$. Using Lemma 2.11 , it follows that $\Delta^{\prime}$ is inner by $g_{B}$. Hence $\Delta$ is inner by $g_{B}+f_{Y}$. Arguing as in the end of the proof of Lemma 2.11, we may obtain the estimate of a generating functional for $\Delta$.

We conclude by extending the result to arbitrary free products.

Theorem 2.12. Let $\left(\mathscr{A}_{\gamma}\right)_{\gamma \in \Gamma}$ be a family of cyclicly amenable Banach algebras, and suppose there is $K>0$ such that, if $\delta_{\gamma}: \mathscr{A}_{\gamma} \rightarrow \mathscr{A}_{\gamma}^{*}(\gamma \in \Gamma)$ are inner derivations, then we can find generators $f_{\gamma} \in \mathscr{A}_{\gamma}^{*}(\gamma \in \Gamma)$ such that $\left\|f_{\gamma}\right\| \leqq K\left\|\delta_{\gamma}\right\|$. Then $\underset{\gamma \in \Gamma}{\hat{\bullet}} \mathscr{A}_{\gamma}$ is cyclicly amenable.

Proof. Let $\Delta: \underset{y \in \Gamma}{\hat{\bullet}} \mathscr{A}_{\gamma} \rightarrow\left(\underset{\gamma \in \Gamma}{\hat{\bullet}} \mathscr{A}_{\gamma}\right)^{*}$ be a cyclic derivation. Let $\mathscr{P}_{0}(\Gamma)$ be the net of finite subsets of $\Gamma$ ordered by inclusion and let $i_{E}: \mathscr{A}_{\gamma} \rightarrow \underset{\gamma \in \Gamma}{\hat{\bullet}} \mathscr{A}_{\gamma}\left(E \in \mathscr{P}_{0}(\Gamma)\right)$ be the canonical embeddings. Since

$$
\left(\mathscr{A}_{\gamma_{1}} \hat{\bullet} \cdots \hat{\bullet} \mathscr{A}_{\gamma_{n}}\right) \cong\left(\mathscr{A}_{\gamma_{1}} \hat{\bullet} \cdots \hat{\bullet} \mathscr{A}_{\gamma_{n-1}}\right) \hat{\bullet} \mathscr{A}_{\gamma_{n}}
$$

$(n \geqq 2)$ we have inductively, using the assumption, that $\underset{y \in E}{\bullet} \mathscr{A}_{\gamma}\left(E \in \mathscr{P}_{0}(\Gamma)\right)$ are cyclicly amenable. For $E \in \mathscr{P}_{0}(\Gamma)$ let $f_{E} \in\left(\underset{\gamma \in \Gamma}{\hat{\bullet}} \mathscr{A}_{\gamma}\right)^{*}$ be an extension of a generator of the (inner) derivation $i_{E}^{*} \Delta i_{E}$. Using the estimate in Proposition 2.8, the hypothesis ensures that we can arrange that $\sup \left\{\left\|f_{\mathbf{E}}\right\| E \in \mathscr{P}_{0}(\Gamma)\right\}<\infty$. If $f$ is a $w^{*}$-limit point of a subnet of $\left(f_{E}\right)_{E \in P o(\Gamma)}$ then $f$ is a generator for $\Delta$.

Corollary 2.13. Every Banach algebra $\mathscr{A}$ is the quotient of a cyclicly amenable Banach algebra, which may be taken to be separable, if $\mathscr{A}$ is.

Proof. One checks easily that $l^{1}(\mathbf{N})$ is cyclicly amenable. Let $X$ be any set. From Theorem 2.12 it follows that, if $\mathbf{F}_{X}$ is the free semigroup on $X$, then $l^{1}\left(\mathbf{F}_{\mathbf{X}}\right)$ is cyclicly 
amenable. It is folklore that any Banach algebra $\mathscr{A}$ is a quotient of $l^{1}\left(\mathbf{F}_{S}\right)$, where $S$ is a dense subset of the unit ball in $\mathscr{A}$.

Acknowledgement. This work was completed while the author was a guest at CMA, Australian National University. He is grateful for the Visiting Fellowship that made the stay possible.

\section{REFERENCES}

1. W. G. Bade, P. C. Curtis, Jr. and H. G. Dales, Amenability and weak amenability for Beurling and Lipschitz algebras, Proc. London Math. Soc. 57 (1987), 359-377.

2. E. Christensen, E. G. Efrros and A. M. Sinclair, Completely bounded multilinear maps and $C^{*}$-algebraic cohomology, Invent. Math. 90 (1987), 279-296.

3. A. Connes, Non-commutative differential geometry, Inst. Hautes Etudes Sci. Publ. Math. 62 (1985), 41-144.

4. B. L. Feigin and B. L. Tzygan, Additive $K$-theory, in K-theory, Arithmetic and Geometry (Lecture Notes in Math. 1289, Springer-Verlag, 1987), 67-209.

5. N. Grønbaek, A characterisation of weak amenability, Studia Math. 94 (1989), 149-162.

6. U. HaAgerup, The Grothendieck inequality for bilinear forms on $C^{*}$-algebras, Adv. in Math. 56 (1985), 93-116.

7. U. HaAgeruP, All nuclear $C^{*}$-algebras are amenable, Invent. Math. 74 (1983), 305-319.

8. B. E. Johnson, Derivations from $L^{1}(G)$ into $L^{1}(G)$ and $L^{\infty}(G)$, in Proc. Internat. Conf. on Harmonic Analysis, Luxembourg, 1987 (Lecture Notes in Math., Springer-Verlag), to appear.

9. B. E. Johnson, Weak amenability of group algebras, Preprint, 1990.

10. A. M. Sinclair, Continuous semigrous in Banach algebras, (London Math. Soc. Lecture Notes 63, Cambridge University Press, Cambridge, 1982).

11. B. L. Tzygan, Homology of matrix Lie algebras over rings and Hochschild homology, Uspekhi Mat. Nauk 38 (1983), 217-218.

Københavns Universitets Matematiske Institut

UNIVERSITETSPARKEN 5

DK-2100 KøBENHAVN $\varnothing$

DenMark 